Int. J. Dev. Biol. 62: 605-612 (2018)

https://doi.org/10.1387/ijdb.180118as

\title{
Nerve roles in blastema induction and pattern formation in limb regeneration
}

\author{
AKIRA SATOH*, KAZUMASA MITOGAWA and AKI MAKANAE \\ Okayama University, Research Core for Interdisciplinary Sciences (RCIS), Kitaku, Okayama, Japan
}

\begin{abstract}
Compared to amniotes, amphibians are widely known to be great healers. Urodele amphibians in particular have tremendous regeneration abilities, and can even regenerate organs, such as the brain, the heart and the limbs. Limb regeneration, in particular, has been investigated since it is representative of their superior regeneration abilities, and the presence of nerves has been examined in detail because they play essential roles in limb regeneration. Without nerves, there is no regeneration. Recent research has succeeded in outlining nerve regulation in the early phases, namely, the blastema induction phase. Based on the results of a few classic studies, it was believed that nerves played minimal roles in the later phases. In the present review, we first summarize the recent insights into the roles of nerves in blastema formation, and in the later stages, pattern formation becomes the focus. Pattern formation in limb regeneration has been interpreted in an intercalary manner. Recent findings point to the participation of nerves in the intercalary regulation of limb regeneration. This may change the current thinking on the effects of nerves on pattern formation in limb regeneration. Although the importance of nerves in amphibian limb regeneration has been recognized, the extent of their importance has remained unclear since the nerve entities were undetermined. This ambiguity was a large obstacle to investigating and comparing regeneration abilities in other species. Recent insights into nerves in limb regeneration may help overcome this obstacle and lead to future advancements.
\end{abstract}

KEY WORDS: intercalation, blastema induction, limb regeneration, nerve, organ regeneration

\section{Introduction}

The reconstitution of three-dimensionally complex structures, such as organs, has been a significant challenge, even in this era of biotechnology. Attempts at organ-level reconstitution have been made, and remarkable progress has been achieved. Blastocyst complementation in particular would be considered a breakthrough in the area (Kobayashi et al., 2010). Such a technique would enable the development of an organ in a different species. Although blastocyst complementation would make organ reconstitution possible, the contamination of host cells in an induced organ and the necessity of a sacrifice remain controversial. Many animals can, however, regenerate three-dimensionally complex organs. They do not require the loss of any other life or complex in vitro procedures to regenerate their organs. Investigating these regeneration-competent animals should help us learn to arrange many kinds of stem cells spatio-properly, which could lead to successful three-dimensional organ reconstitution.

Urodele amphibians have been stydied by researchers for their remarkable regeneration abilities. Although mammals essentially have no organ-level regeneration ability, very limited but certain regeneration can be observed in amputated mammalian digit tips (Borgens, 1982, Dolan et al., 2018). Mammals can regenerate the fingertip when a terminal phalange is amputated approximately one-third from the distal end (Han et al., 2005). Proximal level amputation, however, results in no regeneration. It has been widely believed that the organ-level regeneration ability was lost through evolution. Regeneration ability would therefore not be specifically evolved in urodele amphibians, but would represent a retaining of this ability that we lost during our evolution. This may be supported by the fact that many animals do possess organ-level regenera-

Abbreviations used in this paper: BMP, bone morphogenetic protein; FGF, fibroblast growth factor.

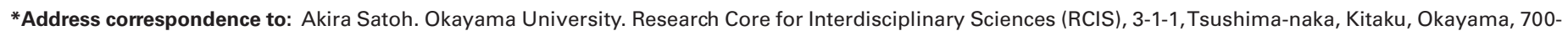
8530, Japan. Tel; +81-86-251-8421. E-mail: satoha@cc.okayama-u.ac.jp
} 
tion abilities. It should be noted that there are a few reports that a species-specific gene (orphan gene) is involved in newt limb regeneration. Prod-1 has been found in salamander families but not yet in other vertebrates (Geng et al., 2015). Prod-1 works as a receptor of Newt anterior gradient ( $n A G)$, which plays important roles in limb regeneration(Kumar et al., 2007). Specifically, nAG was reported as a blastema inducer in newts (Kumar et al., 2007). Despite the obvious activity, blastema induction activity has only been reported in newts, not other species so far. On the other hand, another regulatory mechanism can play a role in blastema induction, which has been conserved in not only newts but also some other amphibians (below). This strongly suggests that the stem of the regulatory network is conserved even though some specific mechanisms may be involved in each species. From this perspective, our genome likely retains a set of regeneration cascades that are suppressed by the genetic cascade acquired in amniote evolution. Otherwise, our genome may have lost a portion of this regeneration cascade, which would result in a loss of regeneration abilities. To identify this regeneration cascade, amphibian limb regeneration is an ideal model, and limb regeneration in amphibians has therefore been investigated as a representative form of regeneration activity.

Amphibian limb regeneration involves at least two processes: 1 ) blastema formation and 2) pattern formation. The former is related to inducing stem cells and the latter is related to coordinating and organizing stem cells to restore a structure. Understanding these two steps is helpful for stem cell research aimed at realizing organ re-creation. This review focuses on the nerve roles and Fgf functions in the above two phases.

\section{Blastema formation and nerve dependence}

Limb regeneration in amphibians always accompanies blastema formation (Fig.1). Regeneration-incompetent animals, including humans, do not have the ability to grow a blastema, which has similar features to a blastema in urodele amphibians, after limb amputation. Thus, limb blastema formation is a key event in successful limb regeneration. A blastema can be recognized within 2 weeks after limb amputation, and consists of many undifferentiated mesenchymal cells and a regeneration-specific epithelium (Fig. 1) (Nye et al., 2003). Blastema mesenchymal cells have been proven to be limb bud-like cells (Muneoka and Bryant, 1982). Although they derive from differentiated tissues, their detailed cell lineage still needs to be further investigated. Blastema cells are undifferentiated cells that are composed of heterogeneous populations, similar to those of developing limb buds (Kragl et al., 2009). Some are unipotent and some are multipotent. Dermis-derived blastema cells can participate in multiple cell lineages (Currie et al., 2016, Hirata et al., 2010, Kragl et al., 2009). The appearance of such multipotent cells is a unique feature of amphibian limb regeneration. The multipotency of dermis-derived blastema cells appears similar to that of limb bud mesenchymal cells. The majority of limb bud cells are derived from the lateral plate mesoderm and have restricted multipotency (Pearse et al., 2007). These cells can differentiate into cell types within connective tissue lineages, such as dermal cells, chondrocytes, and tenocytes. Dermis-derived blastema cells have the same tendency (Currie et al., 2016, Hirata et al., 2010, Kragl et al., 2009). This suggests that dermal fibroblasts return to their cell fate up to limb bud mesenchymal cells. To date, however, there is no molecular evidence of a return to cell fate in dermal fibroblasts. Once a regeneration blastema is established, it can be considered a structure similar to a developing limb bud. A regeneration blastema appears to reuse most of the same genetic cascades as a developing limb bud to generate a patterned limb although the involvement of nerves in limb regeneration processes differs. Importantly, amphibian limb development is not totally unique when compared to amniotes. For instance, Shh is expressed in the posterior side of a developing limb bud in both amphibians and amniotes (Chang et al., 1994, Endo et al., 2000, Nacu et al., 2016, Riddle et al., 1993, Torok et al., 1999). Since the limb developmental processes of amphibians and amniotes are comparable, we will first focus on the blastema induction mechanism in amphibians first.

Nerves have been well recognized as the essential tissue for blastema formation. Todd described the importance of nerves in limb regeneration (Todd, 1823)(Fig. 2). When nerves are removed at the time of, or prior to, amputation, blastema formation is completely prevented. In contrast, if nerves are rerouted to skin wounds, which generally do not regenerate limbs, they can reprogram from skin wound healing to blastema formation (Endo et al., 2004, Makanae
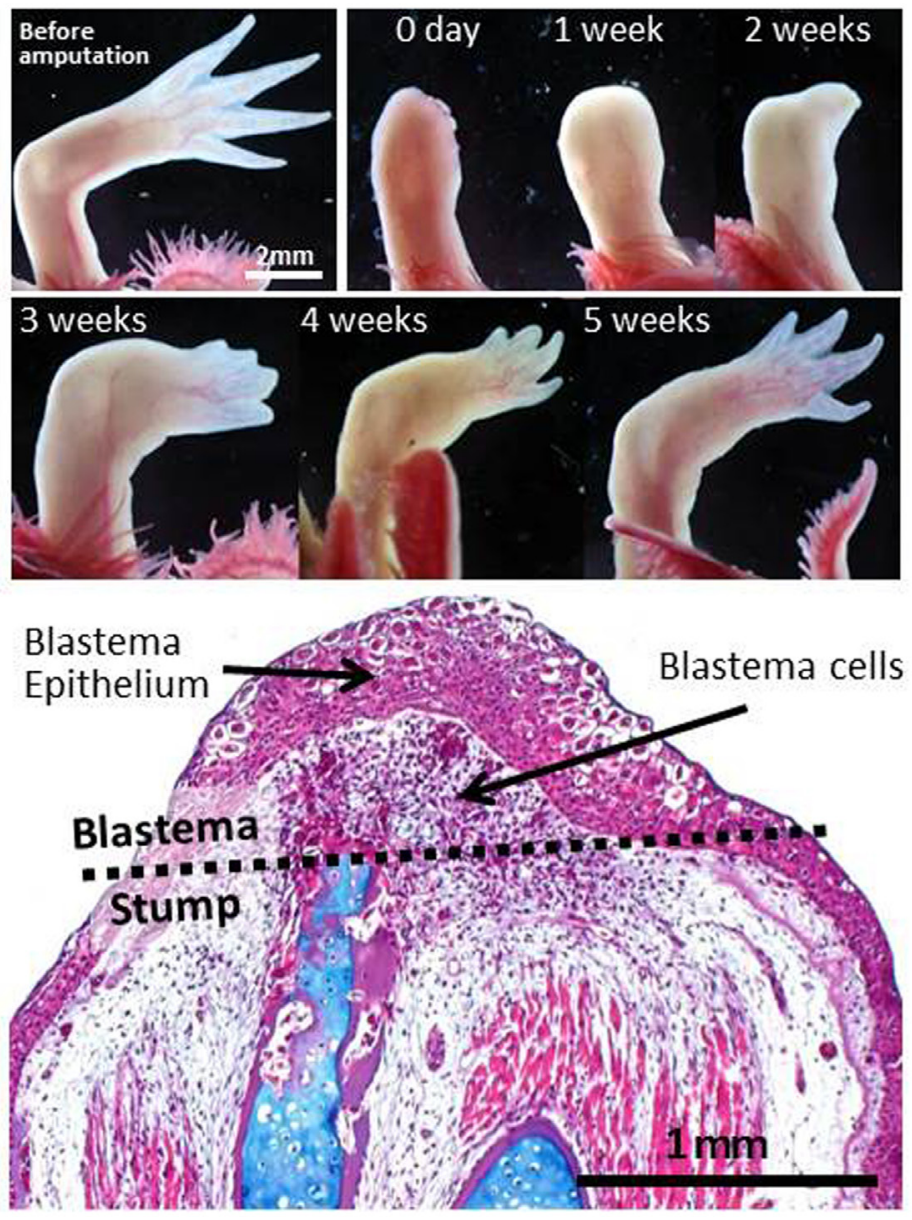

Fig. 1. The process of limb regeneration and a section of a blastema. The upper panels show the normal limb and regenerating limbs. The lower panel shows the section of a regenerating blastema. The section was stained with Alcian blue, hematoxylin and eosin. The dotted line indicates the border of the amputation plane. 


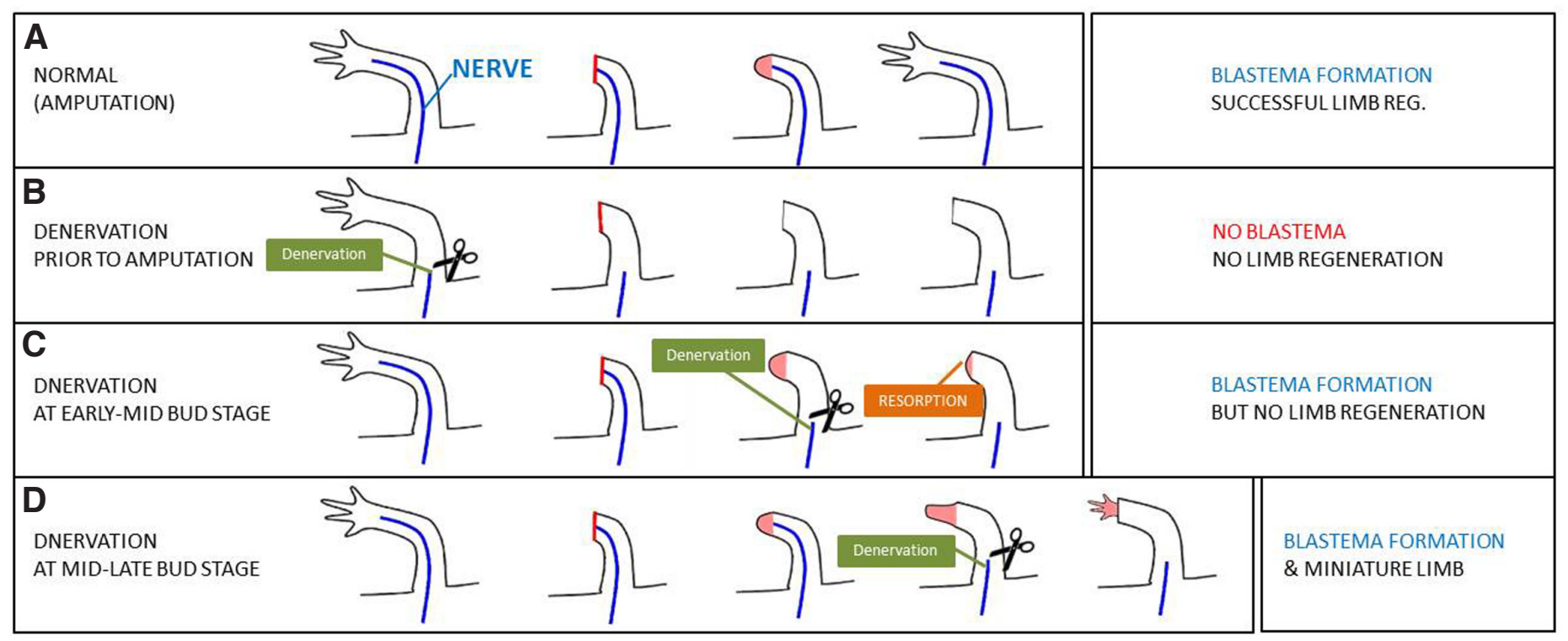

Fig. 2. Summary of denervation effects in limb regeneration. (A) The nerve plays an essential role in successful limb regeneration. (B) Denervation prior to limb amputation results in no blastema formation. (C) Denervation during the early-mid bud stage results in blastema resorption. (D) Denervation during the mid-late bud stage results in blastema resorption or the formation of a miniature limb.

and Satoh, 2012, Satoh et al., 2015)(Fig. 3A). The nerve-induced blastema shows the ability to produce a perfectly patterned limb when the blastema is in the proper condition (Fig. 3A). This suggests that nerves (and wounding) are sufficient and necessary for blastema induction. Nerve molecule entities have been investigated for nearly 200 years, and various candidates have so far been proposed: Glial growth factor 2 (Ggf2), substance P, transferrin, nAG, Neuregulin, Fibroblast growth factors (Fgfs), Bone morphogenetic proteins (Bmps) (Brockes and Kintner, 1986, Farkas et al., 2016, Globus, 1988, Mullen et al., 1996, Munaim and Mescher, 1986, Weis and Weis, 1970). Ggf-2, substance P, and transferrin can promote blastema cell proliferation but do not function in blastema induction (Brockes and Kintner, 1986, Globus, 1988, Munaim and Mescher, 1986). nAG, Neuregulin, and Fgfs+Bmp(s) are the reported nerve factors with blastema inductive abilities (Farkas et al., 2016, Kumar et al., 2007, Makanae et al., 2013). nAG was described above. Neuregulin is expressed in nerves and can serve as a substitute for nerve roles in blastema induction in axolotl limb regeneration (Farkas et al., 2016). Among the reported nerve factors, the combined activity of $\mathrm{Fgf}$ and $\mathrm{Bmp}$ genes can induce a blastema in multiple species (Makanae et al., 2013, Makanae et al., 2014a, Mullen et al., 1996, Satoh et al., 2011, Satoh et al., 2015). Fgf2 was suggested to be involved in nerve regulation in amphibian limb regeneration decades ago (Cannata et al., 2001, Mullen et al., 1996). Fgf2 application alone is, however, insufficient to induce a blastema. Multiple Fgf proteins are likely secreted from nerves to a wound site since the expression of multiple Fgf genes in axolotl neurons has been reported (Christensen et al., 2001, Makanae et al., 2014a, Satoh et al., 2008). Fgf2, Fgf7, Fgf8, and Fgf10 are the genes expressed in axolotl neurons (Makanae et al., 2013, Makanae et al., 2014a, Mullen et al., 1996, Satoh et al., 2008). Fgf-receptor 1 (Fgfr1) for Fgf2 and Fgf8 is expressed in a blastema mesenchyme, and Fgfr2 for Fgf7 and Fgf10 is expressed in the blastema epithelium (Poulin et al., 1993). Fgf7 and Fgf10 have similar functions in the specification of limb bud epidermis during chick limb development (Yonei-Tamura et al., 1999). Hence, Fgf7 and Fgf10 can be expected to play similar roles in the blastema epithelium. Nerves interact with the wound epithelium that covers exposed wound surfaces immediately after wounding, prior to the appearance of blastema mesenchymal cells (Satoh et al., 2008). Nerve presence or Fgf7 application is sufficient to induce a blastema epithelial marker gene, Sp9 (Satoh et al., 2008). However, Fgf7 alone is insufficient to induce a blastema. Ligands for Fgfr1, Fgf2 and Fgf8, are expressed in axolotl dorsal root ganglias (DRGs) and are sufficient to induce a blastema (Makanae et al., 2014a, Satoh et al., 2011). When an Fgf2+Fgf8-soaked bead is grafted to wounded skin, a blastema is induced without apparent nerve presence (Satoh et al., 2011). The Fgf-induced blastema cannot maintain its growth and the induced blastema eventually disappears. Generally, skin from the contralateral side of a limb is critical for growing a well-patterned limb. Even when such essential circumstances are established, the Fgf-induced blastema cannot maintain its growth. Bmp2 or Bmp7 are also expressed in axolotl DRG and the additional supplement of Bmp2 or Bmp7 to the Fgf-induced blastema can maintain the growth, resulting in a well-patterned limb (Fig. 3 B,E,H)(Makanae et al., 2014a). Bmp2 or Bmp7 without Fgfs cannot induce a blastema, which suggests that Bmp plays a role in the maintenance of blastema growth and patterning. This blastema induction by Fgfs+Bmp is not due to the specialty of axolotl Fgfs and Bmps. It is, of course, possible to induce a limb from wounded skin with the skin graft through the electroporation of axolotl Fgf2, Fgf8, and Bmp7 (Fig. 3 C-G). Similarly, using mouse/human recombinant proteins yielded the same results (Fig. $3 \mathrm{H})$. These findings strongly suggest that axolotl Fgfs and Bmp7 are functionally similar to those of other vertebrates. Fgfs can serve as a substitute for nerve function in blastema formation, and Bmp7 and/or Bmp2 signaling is involved in nerve roles in blastema formation in urodele amphibians.

Nerve dependence of limb regeneration was also reported in Xenopus laevis. A Xenopus tadpole can regenerate its limb buds 
in the early stages (Dent, 1962). The regeneration abilities of limb buds gradually decrease as the limb develops. A post-metamorphic froglet still has the ability to raise a blastema even though it cannot form a proper limb. These patterning defects are mentioned below. In the tadpole stages, limb bud regeneration is nerve-independent but Fgf-dependent (Cannata et al., 2001). In the regenerationcompetent stages, Fgf2 is expressed in a limb bud mesenchyme. In the later stages, the Fgf2 expression level decreases and consistent regeneration ability disappears (Cannata et al., 2001). Exogenous Fgf10 application onto an amputation plane can temporarily restore the loss of regeneration abilities (Yokoyama et al., 2000). As for Bmp-signaling, the involvement of Bmp-signaling in tadpole limb bud regeneration has been reported. The overexpression of a transient Noggin, a Bmp-signaling antagonist, is sufficient to prevent the limb regeneration processes (Beck et al., 2006). These Noggin-expressing amputated limb buds fail to regenerate limbs from the early phase of regeneration, suggesting that Bmp-signaling is involved in the early regulation of limb regeneration. As for blastema induction in a froglet limb, nerve dependence is apparent (Endo et al., 2000). Nerve removal from a froglet's limb results in the complete prevention of blastema formation (Endo et al., 2000). The phosphorylation of ERK, which is one of the major downstream cascades of Fgfsignaling, is promoted by the nerve presence in a regenerating blastema (Suzuki et al., 2007). Xenopus DRG express Fgf genes (Mitogawa et al., 2018). Furthermore, Fgf2, Fgf8, and Bmp7 application can induce a blastema from simply wounded skin, similar to axolotls (Satoh et al., 2015). This strongly suggests that Fgf-signaling from nerves plays a major role in blastema induction, even in Xenopus.

Fin regeneration has many similarities to limb regeneration. In fact, pectoral fins have been considered an ancestral structure of forelimbs. Zebrafish, Danio renio, can regenerate their fins after amputation and the regeneration process is nerve dependent (Simoes et al., 2014). The nerve factors, however, have yet to be identified in zebrafish fin regeneration. Denervation prevents blastema formation, and the blastema induction mechanism has been well investigated. In a study focusing on Fgf-signaling, the importance of Fgf-signaling in blastema formation has been shown (Poss et al., 2000). Some Fgf genes are related to fin regeneration, and among them, Fgf20 has been proven to be an essential regulator for blastema formation in zebrafish fin regeneration (Whitehead et al., 2005). Fgf20 expression was downregulated by denervation, suggesting that nerves have positive effects on Fgf20 expression. This is similar to the relationship between nerves and Fgf genes in limb regeneration (Christensen et al., 2001, Endo et al., 2000, Satoh et al., 2017).

The blastema induction mechanism in other vertebrates, including mammals, is still being studied. Limb regeneration in amniotes has been investigated in chick embryos and mouse neonates. It was found that chicken limb buds cannot regenerate from the tiny removal of a distal tip. Fgf2 or Fgf4 application, however, can induce regeneration responses until Hamburger and Hamilton stage 25 (Hamburger and Hamilton, 1951) (Kostakopoulou et al., 1996, Taylor et al., 1994). Limb buds in the later stages cannot respond to exogenous Fgf application. In mice, limb regeneration has been studied with the digit amputation model. Even in mice, the very distal tips of terminal phalanges somehow retain their regeneration abilities (Simkin et al., 2015). Amputation approximately one-third from a distal tip results in full regeneration of terminal phalanges. A slightly more proximal am-
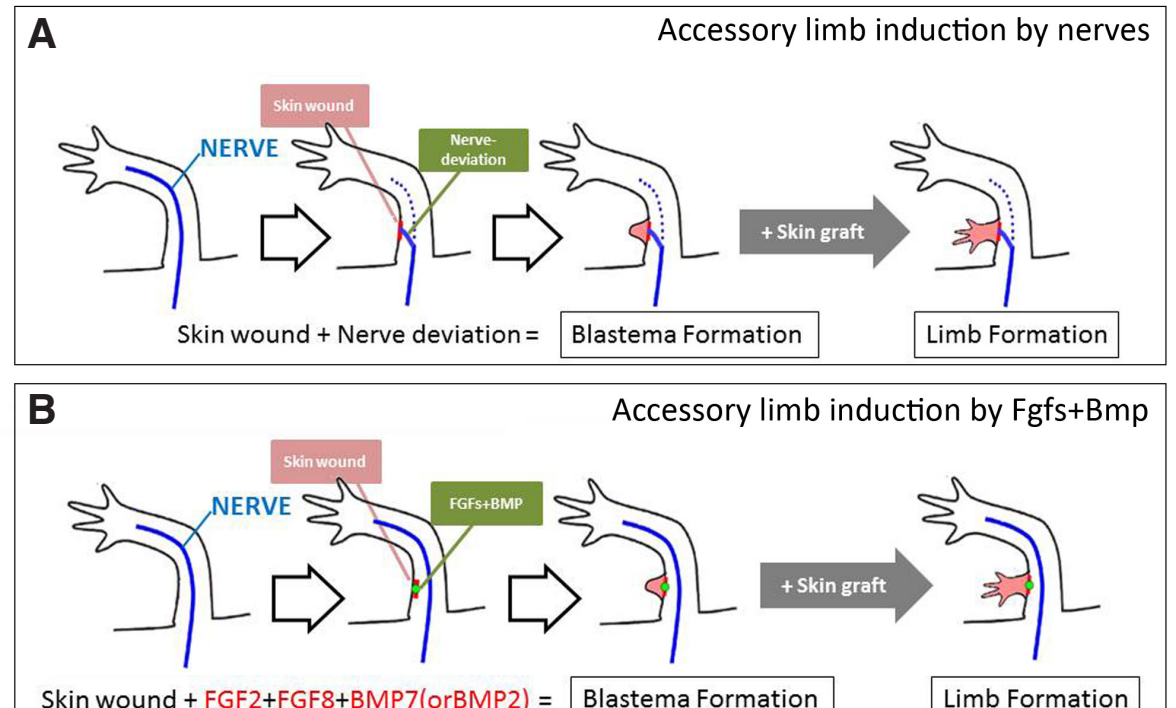

Skin wound + FGF2+FGF8+BMP7(orBMP2) $=$ Blastema Formation $\quad$ Limb Formation

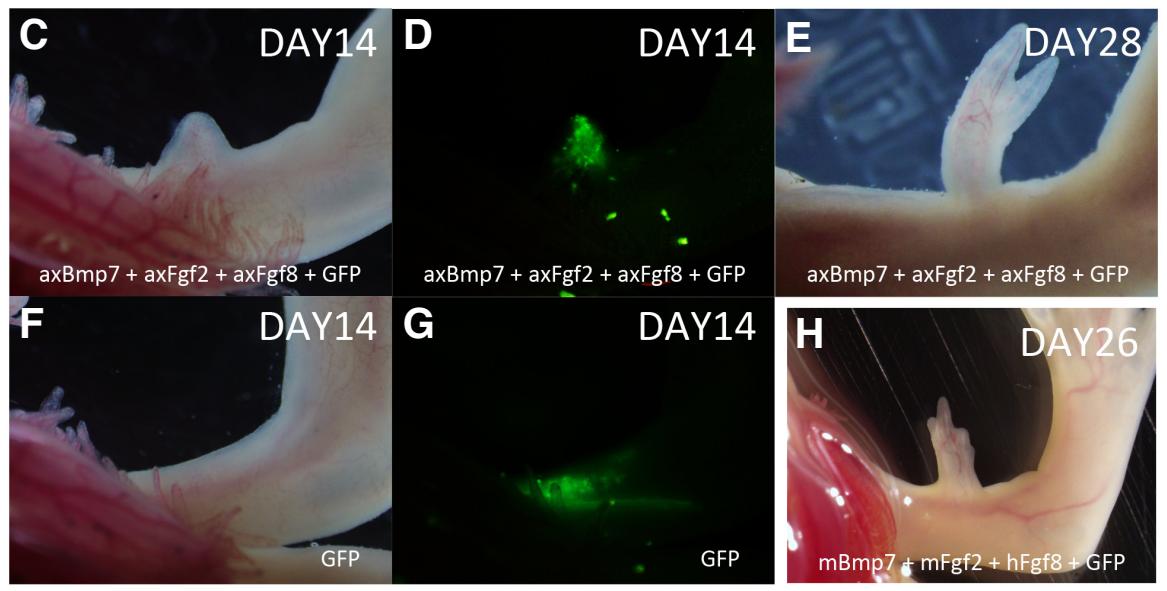

Fig. 3. Summary of the accessory limb model. (A) A blastema can be obtained by skin wounding and nerve deviation. For a perfectly patterned limb, a skin graft from the contralateral side of a limb is necessary. (B) Fgf2+Fgf8+Bmp7 can substitute for nerves in the accessory limb model. Accessory limb induction by mammalian Fgf2 +Fgf8+Bmp7 and axolotl Fgf2 $+F g f 8+B m p 7$. (C-E) Skin was peeled off and a small piece of a skin graft from the contralateral side of the limb was placed prior to the electroporation. Axolotl Fgf2 +Fgf8+Bmp7 plasmids were electroporated into day 3 wounded axolotl skin. The electroporation gave rise to the accessory limb. GFP plasmids were electroporated as an indicator. (F,G) Negative control. GFP plasmids were electroporated under the same conditions and no blastema was induced. (H) Mouse Fgf2, human Fgf8, and mouse Bmp7 proteins were grafted into the wounded axolotl skin, giving rise to the accessory limb. 
putation, however, generally results in no regeneration (Yu et al., 2010). Bmp2 application results in digit regeneration, but it remains unknown whether a blastema similar to a blastema in amphibian limb regeneration is induced. Blastema induction in mouse digit tip regeneration should be carefully assessed with molecular markers in order to compare it with that in amphibians.

It is a curious coincidence that Fgf- and Bmp-signaling are involved in the early phase of regeneration in some animals, although their detailed regulations differ. We need to carefully examine the detailed mechanisms of Fgf- and Bmp-signaling in regeneration in a variety of animals so that we can identify the true conserved regeneration principle.

\section{Nerve roles in patterning in limb regeneration}

Once a blastema forms, it is believed that the induced blastema mostly re-undergoes limb developmental processes to restore a patterned limb. A blastema is similar to a developing limb bud in terms of its features and gene expression pattern. However, an involvement of nerves is one of the significant differences between limb development and regeneration. Nerves do not appear to play a large role in the limb patterning of the limb developmental process. A nerve-free limb bud was demonstrated to develop limb morphology without obvious malformations in a chicken embryo (Hunt, 1932, Lillie, 1904, Murray and Huxley, 1925). Limb buds were isolated from an embryo and allowed to develop on the chorio-allantois. Under these conditions, axons cannot penetrate into the cultured limb bud, resulting in an axon-free limb bud. All nutrients were supplied, and the cultured limb bud developed a limb shape autonomously. These results strongly suggest that nerves do not have a significant function in limb patterning during limb development. As for urodele amphibians, some classic studies suggest the roles of nerves in the limb patterning involved in regeneration. Denervation from a regenerating blastema in the late bud-stage results in a miniature limb (Fig. 2)(Powell, 1969, Schotté and Butler, 1944, Singer and Craven, 1948). This study puts forth the present consensus on nerve functions in the patterning of limb regeneration. However, it is also true that denervation from a blastema in the mid-bud stage resulted in no patterned structure and the regression of the blastema (Schotté and Butler, 1944)(Fig. 2). Similarly, if a blastema in the middle stages is grafted onto a body, and proper innervation from the trunk is prevented, the grafted blastema cannot maintain itself, although a blastema in the later stages can maintain and develop further under the same condition (Faber, 1960, Wallace, 1981). This nerve dependence of a blastema is unexpected based on insights from limb development. The nerve dependence of a regeneration blastema is a specific phenomenon in limb regeneration that we would like to focus on. Denervation after blastema formation was investigated, and it was concluded that it had minimal effect on regeneration (Faber, 1960, Powell, 1969, Schotté and Butler, 1944, Singer and Craven, 1948, Stocum, 1968, Wallace, 1981). Here, we focus on three of these classic representative papers that describe miniature limb formation by denervation (Fig. 2). Schotté and Butler reported that denervation from a blastema in an early stage results in resorption, and that denervation from a blastema in the mid- to late-bud stage resulted in either miniature limbs or resorption in axolotls (Schotté and Butler, 1944)(Fig. 2). Due to the low resolution of the image, it is very hard to determine the blastema stages in the paper. According to their drawings, times, and animal sizes, blastemas do appear to sufficiently reach the mid- to late-bud stages, however. Singer and Graven (1948) also reported similar results in a newt (Singer and Craven, 1948). Denervation performed in the early blastema phases resulted in resorption, and denervation in approximately the mid-bud stage did not allow the blastemas to finish patterning in half of the samples. Powell also reported that denervation in the early-bud stage resulted in no further regeneration (Powell, 1969). The blastema that Powell called the "early bud" was pictured. The sufficiently dome-like shape, numerous blastema cells, and thickened epidermis implies that this blastema is, however, in the mid-bud stage according to the stage table (Iten and Bryant, 1973). It is widely accepted that the time required to reach each specific blastema stage varies depending on conditions such as temperature and animal size. Considering the results of these classic studies, it is very likely that denervation from a blastema till the mid-bud stage severely affects morphogenesis. This was recently confirmed from a slightly different angle. The accessory limb model (ALM) is an alternative experimental system for amphibian limb regeneration (Endo et al., 2004, Endo et al., 2015, Satoh et al., 2007). In this model, limb amputation is no longer necessary. Skin wounding, skin grafting from the contralateral side of a limb, and nerve rerouting are sufficient to induce full limb regeneration, resulting in an accessory limb. Endo et al., reported that denervation after blastema formation resulted in the resorption of a blastema (Endo et al., 2004). Denervation in the ALM is performed on day 10, when Shh, Fgf8, Msx2, Hoxa13, and Hoxd13 are already expressed (Nacu et al., 2016, Satoh et al., 2007). This suggests that limb patterning begins at that stage which, in turn, suggests that nerves play a role in maintaining correct limb patterning processes.

Limb patterning can be interpreted using an intercalation model (Bryant and Gardiner, 2016, Makanae et al., 2014b, Satoh et al., 2010). This conceptual model essentially fills the gap between distal and proximal. It has been hypothesized that distal identity is established first within a blastema. Then, the gap region is induced by interaction between the established distal and stump (proximal) regions. Fgf-signaling plays an important role in this intercalary process. In Xenopus froglet limb development, Fgf8 mediates the intercalary regulation (Shimizu-Nishikawa et al., 2003)(Fig. 4). The presumptive zeugopod portion is removed, and a presumptive stylopod and an autopod are joined together. The removed zeugopod structures are usually not restored at all. When an Fgf8-soaked bead is placed within the space, the removed zeugopod parts are fully restored. Similar observations were reported even in amniotes, Gallus gallus (Fig. 4). Exogenous Fgf2 and Fgf8 applications induce intercalary responses in chicken limb development (Makanae and Satoh, 2018). Even in mammals, it has been suggested that Fgf-signaling mediates the intercalary limb developmental process. It was reported that the zeugopod parts were accordingly decreased as Fgf activity in a limb bud was decreased in mouse limb development (Mariani et al., 2008). This suggests that an Fgf-mediating intercalary mechanism in limb development is conserved among species. It is of note that activation of Fgf-signaling induces intercalary responses in mature axolotl limbs (Satoh et al., 2010)(Fig. 4). A hand part was removed and further amputation was performed in the middle portion of a stylopod in order to excise the entire zeugopod. The isolated hand part was placed onto the amputated stylopod so that there was no zeugopod. This basically gave rise to nothing, as there was no 
restoration of the missing zeugopod (Fig. 4). When Fgf protein(s) was applied at the border, however, apparent restoration of the missing zeugopodial structures could be observed (Fig. 4). In invertebrates, the Fgf-mediating intercalary reaction is implied in planarians. Umesono et al., showed that intercalary regeneration in planarians is regulated by the interaction between Fgf and Wnt signaling (Agata et al., 2014, Umesono et al., 2013). Yet, Fgfr ligands have not been identified in planarians, which suggests that Fgf-signaling mediates intercalary pattern formation in vari-
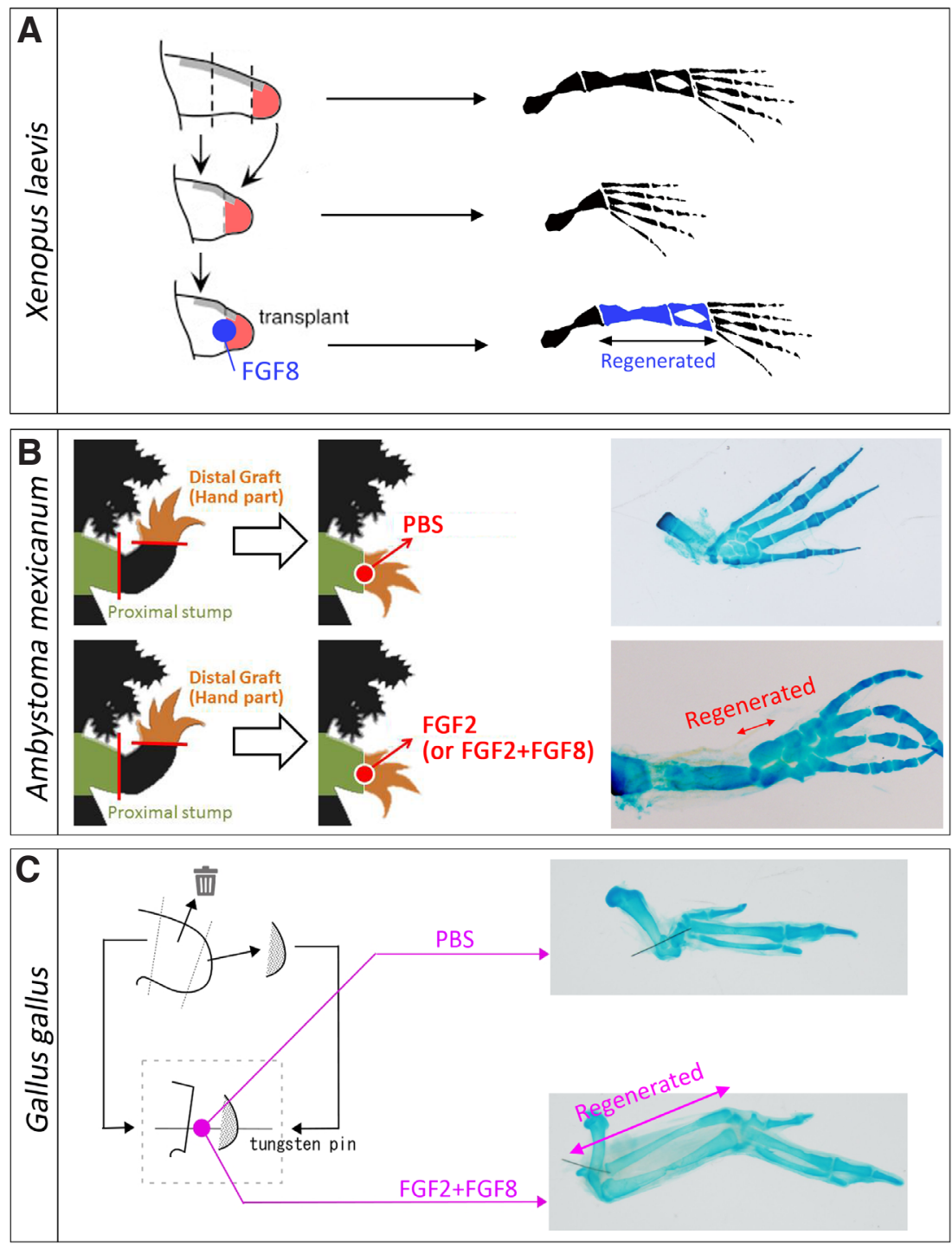

Fig. 4. Intercalary responses in the Xenopus limb bud, axolotl limb, and chick limb bud. (A) Xenopus intercalary limb regeneration. Excision of presumptive lower limb parts (zeugopod) from a developing limb bud results in a loss of the lower limb structure. Ectopic Fgf8 application rescues the lower limb structures. (B) Axolotl intercalary limb regeneration. When a zeugopod is excised and an autopod and stylopod are joined together, no restoration of a zeugopod can be observed. Ectopic Fgf application results in the regeneration of the missing zeugopod. (C) Chick intercalation limb regeneration response. A presumptive lower wing part is dissecting out and a distal part (a presumptive autopod) is directly placed onto a proximal part (a presumptive stylopod). When a PBS-soaked bead is placed in the border of the graft, no zeugopod structures are regenerated. When an Fgf2+Fgf8 soaked bead is placed in the same location, the removed zeugopod parts are regenerated. us situations.

Nerves are expected to be a source of Fgfs in limb regeneration. axolotls and Xenopus froglets (Christensen et al., 2001, Endo et al., 2000, Mullen et al., 1996, Satoh et al., 2017, Yokoyama et al., 2000). Thus, nerves likely contribute to the creation of an Fgf-enriched environment in a regeneration blastema. Fgf-enriched blastemas require ideal conditions to achieve intercalary regulations. Denervation may cause the loss of this Fgf-enriched field, resulting in the suspended growth or resorption of a blastema. The relationship between nerve-related Fgf-enriched blastema fields and intercalary regulation remains unclear. One experiment on Xenopus froglet limb regeneration speculates on the regenerative environment by nerves (Mitogawa et al., 2018). When nerves are rerouted from a hind limb to a forelimb, these hyperinnervated forelimbs exhibited superior regeneration. Xenopus froglet blastemas generally form a pattern-less cone-shaped cartilaginous structure called a spike (Dent, 1962, Satoh et al., 2005). Hyperinnervated froglet blastemas resulted in a relatively patterned regenerate but still not a perfectly patterned limb. This regenerate improvement indicates that some gene expressions were restored. In a regular Xenopus froglet blastema, Shh is not expressed (Endo et al., 2000, Yakushiji et al., 2007). Hyperinnervation upregulates Shh in a proximal blastema, where $S h h$ is to be expressed. $L m \times 1 b$ is a dorsal regulatory transcriptional factor, and no $L m \times 1 b$ expression is observed in a froglet blastema (Matsuda et al., 2001). Hyperinnervation induces Lmx1b expression in a dorsal portion of a blastema (Mitogawa et al., 2018). These findings enable us to hypothesize that nerves secreting Fgfs create a suitable environment for intercalary regulations involving propergene expression in appropriate areas. Although it is a fascinating hypothesis, no concrete evidence has been shown to date. The fact that nerves secrete Fgfs and that Fgfs mediate intercalary pattern formation strongly suggests that nerves play a role in pattern formation in limb regeneration.

Evidence of nerve-dependent pattern formation has not been satisfactory. As mentioned in this review, however, pattern formation in limb regeneration is likely related to nerve presence. As mentioned above, denervation prevents Fgf8 expression in blastemas (Christensen et al., 2001, Satoh et al., 2017), suggesting a maintenance role for the nerve in terms of Fgf8 expression in a blastema mesenchyme. Loss of Fgf8 expression in a regenerating blastema results in a halt in regeneration, no patterning and resorption (Nacu et al., 2016). These findings suggest that the Fgf-enriched environment created by nerves is important for cell proliferation and pattern formation. 
Cell proliferation and pattern formation are likely very closely related (Bryant and Gardiner, 2016, Stocum, 2011). The primary role of nerves in the later phases is currently thought to be maintaining the proliferation of blastema mesenchyme. Denervation causes a loss of cell proliferation, which results in suspended growth or resorption of a blastema. In contrast, denervation may disrupt intercalary regulations, causing a loss of cell proliferation.

\section{Prospective role of nerves in the limb regeneration of amniotes}

Discovering new medicine is an overarching goal, and researchers in the limb regeneration field consider and discuss this topic. To date, amphibian limb regeneration research has not yet contributed significantly to new medical treatment. Recently, however, the molecules responsible for blastema induction have been revealed. Furthermore, the mechanism of pattern formation is beginning to be revealed, as shown above. Given the mouse digit regeneration and the induction of chicken intercalary responses by Fgfs, it is not unreasonable to believe that the basic genetic set of regeneration mechanisms may exist in our genome. Careful investigation could lead to novel ways in which to cure human wounds.

\section{Acknowledgements}

We thank Dr. Tetsuya Endo for his valuable comments regarding the classical studies. This study was supported by the Naito-Foundation, Grants-in-Aid for Scientific Research (B) \#17H03685, Grants-in-Aid for Challenging Exploratory Research \#17K19400, and the Japan Agency for Medical Research and Development (AMED).

\section{References}

AGATA, K., TASAKI, J., NAKAJIMA, E. and UMESONO, Y. (2014). Recent identification of an ERK signal gradient governing planarian regeneration. Zoology (Jena) 117: $161-162$

BECK, C.W., CHRISTEN, B., BARKER, D. and SLACK, J.M. (2006). Temporal requirement for bone morphogenetic proteins in regeneration of the tail and limb of Xenopus tadpoles. Mech Dev 123: 674-688.

BORGENS, R.B. (1982). Mice regrow the tips of their foretoes. Science 217: 747-50.

BROCKES, J.P. and KINTNER, C.R. (1986). Glial growth factor and nerve-dependent proliferation in the regeneration blastema of Urodele amphibians. Cell45:301-306.

BRYANT, S.V. and GARDINER, D.M. (2016). The relationship between growth and pattern formation. Regeneration 3: 103-122.

CANNATA, S.M., BAGNI, C., BERNARDINI, S., CHRISTEN, B. and FILONI, S. (2001). Nerve-independence of limb regeneration in larval Xenopus laevis is correlated to the level of fgf-2 mRNA expression in limb tissues. Dev Biol 231: 436-446.

CHANG, D.T., LOPEZ, A., VON KESSLER, D.P., CHIANG, C., SIMANDL, B.K., ZHAO, R., SELDIN, M.F., FALLON, J.F. and BEACHY, P.A. (1994). Products, genetic linkage and limb patterning activity of a murine hedgehog gene. Development 120: 3339-3353.

CHRISTENSEN, R.N., WEINSTEIN, M. and TASSAVA, R.A. (2001). Fibroblast growth factors in regenerating limbs of Ambystoma: cloning and semi-quantitative RTPCR expression studies. J Exp Zool 290: 529-540.

CURRIE, JOSHUA D., KAWAGUCHI, A., TRASPAS, RICARDO M., SCHUEZ, M., CHARA, O. and TANAKA, ELLY M. (2016). Live Imaging of Axolotl Digit Regeneration Reveals Spatiotemporal Choreography of Diverse Connective Tissue Progenitor Pools. Dev. Cell 39: 411-423.

DENT, J.N. (1962). Limb regeneration in larvae and metamorphosing individuals of the South African clawed toad. J. Morphol. 110: 61-77.

DOLAN, C.P., DAWSON, L.A. and MUNEOKA, K. (2018). Digit Tip Regeneration: Merging Regeneration Biology with Regenerative Medicine. Stem Cells Transl
Med 7: 262-270.

ENDO, T., BRYANT, S.V. and GARDINER, D.M. (2004). A stepwise model system for limb regeneration. Dev Biol 270: 135-145.

ENDO, T., GARDINER, D.M., MAKANAE, A. and SATOH, A. (2015). The accessory limb model: an alternative experimental system of limb regeneration. Methods Mol Biol. 2015; 1290:101-13. doi: 10.1007/978-1-4939-2495-0_8.

ENDO, T., TAMURA, K. and IDE, H. (2000). Analysis of gene expressions during Xenopus forelimb regeneration. Dev Biol 220: 296-306.

FABER, J. (1960). An experimental analysis of regional organization in the regenerating fore limb of the axolotl (Ambystoma mexicanum). Arch Biol (Liege) 71: 1-72.

FARKAS, J.E., FREITAS, P.D., BRYANT, D.M., WHITED, J.L. and MONAGHAN, J.R. (2016). Neuregulin-1 signaling is essential for nerve-dependent axolotl limb regeneration. Development 143: 2724-2731.

GENG, J., GATES, P.B., KUMAR, A., GUENTHER, S., GARZA-GARCIA, A., KUENNE, C., ZHANG, P., LOOSO, M. and BROCKES, J.P. (2015). Identification of the orphan gene Prod 1 in basal and other salamander families. EvoDevo 6: 9.

GLOBUS, M. (1988). A neuromitogenic role for substance P in urodele limb regeneration. Okada Printing and Publishing.

HAMBURGER, V. and HAMILTON, H.L. (1951). A series of normal stages in the development of the chick embryo. J Morphol 88: 49-92.

HAN, M., YANG, X., TAYLOR, G., BURDSAL, C.A., ANDERSON, R.A. and MUNEOKA, K. (2005). Limb regeneration in higher vertebrates: developing a roadmap. Anat Rec B New Anat 287: 14-24.

HIRATA, A., GARDINER, D.M. and SATOH, A. (2010). Dermal fibroblasts contribute to multiple tissues in the accessory limb model. Dev Growth Differ 52: 343-350.

HUNT, E.A. (1932). The differentiation of chick limb buds in chorio-allantoic grafts, with special reference to the muscles. J. Exp. Zool. 62: 57-91.

ITEN, L.E. and BRYANT, S.V. (1973). Forelimb regeneration from different levels of amputation in the newt,Notophthalmus viridescens: Length, rate, and stages. Wilhelm Roux'Archiv für Entwicklungsmechanik der Organismen 173: 263-282.

KOBAYASHI, T., YAMAGUCHI, T., HAMANAKA, S., KATO-ITOH, M., YAMAZAKI, Y., IBATA, M., SATO, H., LEE, Y.S., USUI, J., KNISELY, A.S. et al., (2010). Generation of rat pancreas in mouse by interspecific blastocyst injection of pluripotent stem cells. Cell 142: 787-799.

KOSTAKOPOULOU, K., VOGEL, A., BRICKELL, P. and TICKLE, C. (1996). 'Regeneration' of wing bud stumps of chick embryos and reactivation of Msx-1 and Shh expression in response to FGF-4 and ridge signals. Mech Dev 55: 119-131.

KRAGL, M., KNAPP, D., NACU, E., KHATTAK, S., MADEN, M., EPPERLEIN, H.H. and TANAKA, E.M. (2009). Cells keep a memory of their tissue origin during axolotl limb regeneration. Nature 460: 60-65.

KUMAR, A., GODWIN, J.W., GATES, P.B., GARZA-GARCIA, A.A. and BROCKES, J.P. (2007). Molecular basis for the nerve dependence of limb regeneration in an adult vertebrate. Science 318: 772-777.

LILLIE, F.R. (1904). Experimental Studies on the Development of Organs in the Embryo of the Fowl (Gallus Domesticus). Biol. Bull. 7: 33-54.

MAKANAE, A., HIRATA, A., HONJO, Y., MITOGAWA, K. and SATOH, A. (2013). Nerve independent limb induction in axolotls. Dev Biol 381: 213-226.

MAKANAE, A., MITOGAWA, K. and SATOH, A. (2014a). Co-operative Bmp- and Fgf-signaling inputs convert skin wound healing to limb formation in urodele amphibians. Dev Biol 396: 57-66.

MAKANAE, A., MITOGAWA, K. and SATOH, A. (2014b). Implication of two differen regeneration systems in limb regeneration. Regeneration (Oxf) 1: 1-9.

MAKANAE, A. and SATOH, A. (2012). Early regulation of axolotl limb regeneration. Anat Rec (Hoboken) 295: 1566-1574.

MAKANAE, A. and SATOH, A. (2018). Ectopic Fgf signaling induces the intercalary response in developing chicken limb buds. Zoological Lett 4: 8.

MARIANI, F.V., AHN, C.P. and MARTIN, G.R. (2008). Genetic evidence that FGFs have an instructive role in limb proximal-distal patterning. Nature 453: 401-405

MATSUDA, H., YOKOYAMA, H., ENDO, T., TAMURA, K. and IDE, H. (2001). An epidermal signal regulates $L m x-1$ expression and dorsal-ventral pattern during Xenopus limb regeneration. Dev Biol 229: 351-362.

MITOGAWA, K., MAKANAE, A. and SATOH, A. (2018). Hyperinnervation improves Xenopus laevis limb regeneration. Dev Biol 433: 276-286. 
MULLEN, L.M., BRYANT, S.V., TOROK, M.A., BLUMBERG, B. and GARDINER, D.M. (1996). Nerve dependency of regeneration: the role of Distal-less and FGF signaling in amphibian limb regeneration. Development 122: 3487-3497.

MUNAIM, S.I. and MESCHER, A.L. (1986). Transferrin and the trophic effect of neural tissue on amphibian limb regeneration blastemas. Dev Biol 116: 138-142.

MUNEOKA, K. and BRYANT, S.V. (1982). Evidence that patterning mechanisms in developing and regenerating limbs are the same. Nature 298: 369-371.

MURRAY, P.D. and HUXLEY, J.S. (1925). Self-Differentiation in the Grafted Limb-Bud of the Chick. J Anat 59: 379-384.

NACU, E., GROMBERG, E., OLIVEIRA, C.R., DRECHSEL, D. and TANAKA, E.M. (2016). FGF8 and SHH substitute for anterior-posterior tissue interactions to induce limb regeneration. Nature 533: 407-410.

NYE, H.L., CAMERON, J.A., CHERNOFF, E.A. and STOCUM, D.L. (2003). Regeneration of the urodele limb: a review. Dev Dyn 226: 280-294.

PEARSE, R.V., 2ND, SCHERZ, P.J., CAMPBELL, J.K. and TABIN, C.J. (2007). A cellular lineage analysis of the chick limb bud. Dev Biol 310: 388-400.

POSS, K.D., SHEN, J., NECHIPORUK, A., MCMAHON, G., THISSE, B., THISSE, C. and KEATING, M.T. (2000). Roles for Fgf signaling during zebrafish fin regeneration. Dev Biol 222: 347-358.

POULIN, M.L., PATRIE, K.M., BOTELHO, M.J., TASSAVA, R.A. and CHIU, I.M. (1993) Heterogeneity in the expression of fibroblast growth factor receptors during limb regeneration in newts (Notophthalmus viridescens). Development 119: 353-361.

POWELL, J.A. (1969). Analysis of histogenesis and regenerative ability of denervated forelimb regenerates of Triturus viridescens. J. Exp. Zool. 170: 125-147.

RIDDLE, R.D., JOHNSON, R.L., LAUFER, E. and TABIN, C. (1993). Sonic hedgehog mediates the polarizing activity of the ZPA. Cell 75: 1401-1416.

SATOH, A., CUMMINGS, G.M.C., BRYANT, S.V. and GARDINER, D.M. (2010). Regulation of proximal-distal intercalation during limb regeneration in the axolotl (Ambystoma mexicanum). Dev Growth Differ 52: 785-798.

SATOH, A., GARDINER, D.M., BRYANT, S.V. and ENDO, T. (2007). Nerve-induced ectopic limb blastemas in the Axolotl are equivalent to amputation-induced blastemas. Dev Biol 312: 231-244.

SATOH, A., GRAHAM, G.M., BRYANT, S.V. and GARDINER, D.M. (2008). Neurotrophic regulation of epidermal dedifferentiation during wound healing and limb regeneration in the axolotl (Ambystoma mexicanum). Dev Biol 319: 321-335.

SATOH, A., IDE, H. and TAMURA, K. (2005). Muscle formation in regenerating Xenopus froglet limb. Dev Dyn 233: 337-346.

SATOH, A., MAKANAE, A., HIRATA, A. and SATOU, Y. (2011). Blastema induction in aneurogenic state and Prrx-1 regulation by MMPs and FGFs in Ambystoma mexicanum limb regeneration. Dev Biol 355: 263-274.

SATOH, A., MAKANAE, A., NISHIMOTO, Y. and MITOGAWA, K. (2016). FGF and BMP derived from dorsal root ganglia regulate blastema induction in limb regeneration in Ambystoma mexicanum. Dev Biol 417: 114-125.

SATOH, A., MITOGAWA, K. and MAKANAE, A. (2015). Regeneration inducers in limb regeneration. Dev Growth Differ 57: 421-429.

SATOH, A., MITOGAWA, K., SAITO, N., SUZUKI, M., SUZUKI, K.T., OCHI, H. and MAKANAE, A. (2017). Reactivation of larval keratin gene (krt62.L) in blastema epithelium during Xenopus froglet limb regeneration. Dev Biol 432: 265-272.

SCHOTT, O. and BUTLER, E. (1944). Phases in regeneration of the urodele limb and their dependence upon the nervous system. J. Exp. Zool. 97: 95-121.

SHIMIZU-NISHIKAWA, K., TAKAHASHI, J. and NISHIKAWA, A. (2003). Intercalary and supernumerary regeneration in the limbs of the frog, Xenopus laevis. Dev Dyn 227: 563-572.

SIMKIN, J., SAMMARCO, M.C., DAWSON, L.A., SCHANES, P.P., YU, L. and MUNEOKA, K. (2015). The mammalian blastema: regeneration at our fingertips. Regeneration (Oxf) 2: 93-105.

SIMOES, M.G., BENSIMON-BRITO, A., FONSECA, M., FARINHO, A., VALERIO, F., SOUSA, S., AFONSO, N., KUMAR, A. and JACINTO, A. (2014). Denervation impairs regeneration of amputated zebrafish fins. BMC Dev Biol 14: 49.

SINGER, M. and CRAVEN, L. (1948). The growth and morphogenesis of the regenerating forelimb of adult Triturus following denervation at various stages of development. J. Exp. Zool. 108: 279-308.

STOCUM, D.L. (1968). The urodele limb regeneration blastema: A self-organizing system: II. Morphogenesis and differentiation of autografted whole and fractional blastemas. Dev Biol 18: 457-480.

STOCUM, D.L. (2011). The role of peripheral nerves in urodele limb regeneration. Eur J Neurosci 34: 908-916.

SUZUKI, M., SATOH, A., IDE, H. and TAMURA, K. (2007). Transgenic Xenopus with prx1 limb enhancer reveals crucial contribution of MEK/ERK and PI3K/AKT pathways in blastema formation during limb regeneration. Dev Bio/304: 675-686.

TAYLOR, G.P., ANDERSON, R., REGINELLI, A.D. and MUNEOKA, K. (1994). FGF-2 induces regeneration of the chick limb bud. Dev Biol 163: 282-284.

TODD, T. (1823). On the process of reproduction of the members of the aquatic salamander. Quart. J. Sci. Lit. Arts 16: 84-96.

TOROK, M.A., GARDINER, D.M., IZPISUA-BELMONTE, J.C. and BRYANT, S.V (1999). Sonic hedgehog (shh) expression in developing and regenerating axolot limbs. J Exp Zool 284: 197-206.

UMESONO, Y., TASAKI, J., NISHIMURA, Y., HROUDA, M., KAWAGUCHI, E., YAZAWA, S., NISHIMURA, O., HOSODA, K., INOUE, T. and AGATA, K. (2013). The molecular logic for planarian regeneration along the anterior-posterior axis. Nature 500: 73-76.

WALLACE, H. (1981). Vertebrate limb regeneration. John Wiley \& Sons., New York.

WEIS, J.S. and WEIS, P. (1970). The effect of nerve growth factor on limb regeneration in Ambystoma. J Exp Zool 174: 73-78.

WHITEHEAD, G.G., MAKINO, S., LIEN, C.L. and KEATING, M.T. (2005). fgf20 is essential for initiating zebrafish fin regeneration. Science 310: 1957-1960.

YAKUSHIJI, N., SUZUKI, M., SATOH, A., SAGAI, T., SHIROISHI, T., KOBAYASHI, H., SASAKI, H., IDE, H. and TAMURA, K. (2007). Correlation between Shh expression and DNA methylation status of the limb-specific Shh enhancer region during limb regeneration in amphibians. Dev Biol 312: 171-182.

YOKOYAMA, H., YONEI-TAMURA, S., ENDO, T., IZPISUABELMONTE, J.C., TAMURA, K. and IDE, H. (2000). Mesenchyme with fgf-10 expression is responsible for regenerative capacity in Xenopus limb buds. Dev Biol 219: 18-29.

YONEI-TAMURA, S., ENDO, T., YAJIMA, H., OHUCHI, H., IDE, H. and TAMURA K. (1999). FGF7 and FGF10 directly induce the apical ectodermal ridge in chick embryos. Dev Biol 211: 133-143.

YU, L., HAN, M., YAN, M., LEE, E.C., LEE, J. and MUNEOKA, K. (2010). BMP sig naling induces digit regeneration in neonatal mice. Development 137: 551-559. 
Further Related Reading, published previously in the Int. J. Dev. Biol.

The evolution of regeneration - where does that leave mammals?

Malcolm Maden

Int. J. Dev. Biol. (2018) 62: 369-372

An integrative framework for salamander and mouse limb regeneration

Duygu Payzin-Dogru and Jessica L. Whited

Int. J. Dev. Biol. (2018) 62: 393-402

Proteomics analysis of regenerating amphibian limbs: changes during the onset of regeneration

Michael W. King, Anton W. Neff and Anthony L. Mescher

Int. J. Dev. Biol. (2009) 53: 955-969

Vertebrate limb regeneration and the origin of limb stem cells

Susan V Bryant, Tetsuya Endo and David M Gardiner

Int. J. Dev. Biol. (2002) 46: 887-896

http://www.intjdevbiol.com/web/paper/12455626

Molecular mechanisms in the control of limb regeneration: the role of homeobox genes D M Gardiner and S V Bryant

Int. J. Dev. Biol. (1996) 40: 797-805

http://www.intjdevbiol.com/web/paper/8877453

Experimental evidence for FGF-1 control of blastema cell proliferation during limb regeneration of the amphibian Pleurodeles waltl.

C Zenjari, Y Boilly-Marer, X Desbiens, M Oudghir, H Hondermarck and B Boilly

Int. J. Dev. Biol. (1996) 40: 965-971

5 yr ISI Impact Factor $(2016)=2.421$
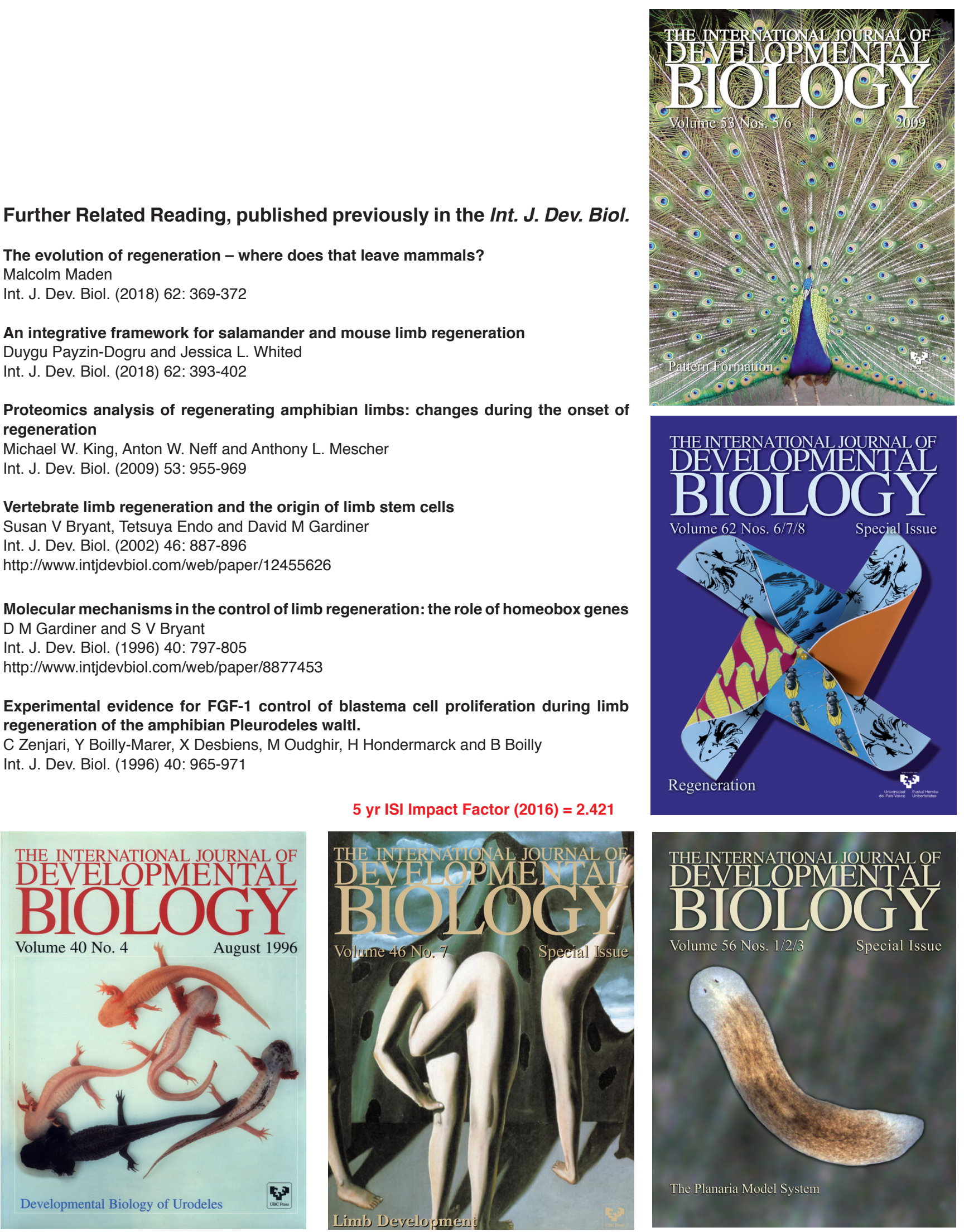